\title{
GALEX catalogs of UV sources: statistical properties and sample science applications: hot white dwarfs in the Milky Way
}

\author{
L. Bianchi $\cdot$ J. Herald $\cdot$ B. Efremova $\cdot$ L. Girardi • \\ A. Zabot · P. Marigo - A. Conti - B. Shiao
}

Received: 20 October 2010 / Accepted: 23 December 2010 / Published online: 18 January 2011

(C) The Author(s) 2011. This article is published with open access at Springerlink.com

\begin{abstract}
We describe the content and properties of UV source catalogs from GALEX's All-Sky Imaging Survey (AIS, $5 \sigma$ depth $\approx 19.9$ (FUV)/20.8(NUV) mag, in the AB system) and Medium-depth Imaging Survey (MIS, $5 \sigma$ depth $\approx 22.6(\mathrm{FUV}) / 22.7$ (NUV) mag), constructed by Bianchi L., et al.: Mon. Not. R. Astron. Soc. (2010, in press). The catalogs contain 65.3/12.6 million (AIS/MIS) unique UV sources with photometric error in NUV less than $0.5 \mathrm{mag}$, over 21435 (AIS)/1579(MIS) square degrees. Matched optical data from GSC-II provide additional $B, R, I$ photometry for the brightest sources, and SDSS provides u g r $i$ z photometry over 7325(AIS)/1103(MIS) square degrees overlap areas. We discuss statistical properties that are relevant for understanding sample selection biases and completeness, in potential science applications of these catalogs. The FUV (1344-1786 ̊) and NUV (1771-2831 ̊) photometry uniquely enable selection of the hottest stellar objects, in particular hot white dwarfs (WD), which are elusive at optical wavelengths because of their hot temperatures and faint luminosities. From the GALEX-SDSS matched sources we
\end{abstract}

L. Bianchi $(\bowtie) \cdot$ J. Herald · B. Efremova

Department of Physics \& Astronomy, Johns Hopkins University, Baltimore, MD, USA

e-mail: bianchi@pha.jhu.edu

L. Girardi

INAF-Padua Observatory, Padua, Italy

A. Zabot

Universidade Federal da Fronteira Sul, Santa Catarina, Brazil

P. Marigo

Department of Astronomy, Padua University, Padua, Italy

A. Conti · B. Shiao

Space Telescope Science Institute, Baltimore, MD, USA selected $\sim 40000$ Milky Way (MW) stars hotter than about $18000 \mathrm{~K}$ (FUV-NUV <-0.13). Their density increases towards low Galactic latitudes, but drops in the MW disk due to dust extinction. The hot-WD density at different Galactic latitudes, analyzed with Milky Way models, constrains the Initial-Final Mass Relation (IFMR), relevant for understanding the yield of chemical elements from intermediate-mass stars and the chemical enrichment of the Galaxy.

Keywords Astronomical Data Bases: catalogues - Stars: white dwarfs - Stars: evolution - Galaxy: stellar content · Ultraviolet: stars · Galaxies: Milky Way

\section{Introduction}

A new paradigm for astrophysical research in recent decades, leading to significant discoveries, has come from extensive surveys at all wavelengths, made possible by large collecting-area ground-based telescopes, advances in largeformat, efficient detectors, and the possibility of mining huge databases with versatile browsing tools. While current and future ground-based surveys (e.g. SDSS, Pan-STARRS, LSST), as in most other wavelength domains, improve on previous catalogs, the space Ultraviolet regime remained basically unexplored in survey mode until the launch of GALEX (Galaxy Evolution Explorer, Martin et al. 2005) in 2003. Over the last five years, GALEX has filled this gap, significantly widening the discovery space in multiwavelength studies, and uniquely enabling selection and characterization of some classes of objects, and discovery of new classes, elusive or undistiguishable at other wavelengths.

We describe current GALEX catalogs of UV sources, and illustrate some of their properties that will aid selection of 
samples for varied purposes by the astrophysical community. Such UV source catalogs are a resource for a number of science investigations, as well as for planning follow-up programs with instruments at any wavelengths and especially with currently available UV instruments (e.g. HST STIS and COS, WFC3) and future UV missions on large scale (e.g. WSO, Shustov et al. 2009) and small scale like UVIT on ISRO Astrosat, rocket flights (e.g. McCandliss et al. 2005) and long-duration-flight ballons.

\section{The GALEX Ultraviolet Sky Surveys. Coverage and characteristics}

GALEX is providing wide-field $\left(1.2^{\circ}\right.$ diameter $)$ imaging in two Ultraviolet (UV) bands simultaneously: FUV $\left(\lambda_{\text {eff }}=1539 \AA, \Delta \lambda=1344-1786 \AA\right)$ and NUV $\left(\lambda_{\text {eff }}=\right.$ $2316 \AA, \Delta \lambda=1771-2831 \AA$ ), with a resolution of 4.2/5.3" (FUV/NUV) (Morrissey et al. 2007). GALEX performs nested surveys with differing sky coverage and depth (see Bianchi 2009 for a summary). Here we describe the source content of the two most extended (over sky area) surveys, the AIS (All-sky imaging survey) and MIS (Medium-depth Imaging survey). Deeper surveys extend the space volume, by reaching distant objects, but are necessarily more restricted in area coverage. GALEX was launched in April 2003 and operated with two detectors until 2009. Currently (2010) the FUV detector is non-operational, and the sky surveys are continuing in the NUV band.

The GALEX data are publicly available through MAST (www.galex.stsci.edu); presently the sixth public data release (GR6) is under way. From the MAST archive, one can download both calibrated images and catalogs of pipeline extracted photometric sources (see Conti et al. 2011 this book, for a description of the GALEX database). In this paper we discuss catalogs of 'unique' GALEX sources from data release \#5 (GR5), which is entirely available and includes most observations with both FUV and NUV detectors, in order to illustrate effects of color (FUV-NUV) sample selections and error cuts, and the statistical properties of the UV sky (initially explored by Bianchi et al. 2005, 2007, 2009a, 2009b, 2010; Bianchi 2009).

\subsection{Catalogs of UV sources}

Figure 1 shows the sky coverage of GALEX's AIS and MIS surveys for GR5, in Galactic coordinates. In this paper we analyze the catalogs constructed by Bianchi et al. (2010) from the GALEX GR5 database. We refer to that paper for extensive details and for technical caveats, useful to those who may want to construct similar catalogs. Here we only briefly summarize how the catalogs were constructed, in order to discuss properties not previously addressed, that are of general interest for scientific applications of these catalogs. ${ }^{1}$

The MAST GALEX database includes all observations, and a source may have multiple measurements if it lies on overlapping areas of contiguous fields, or when the observations are repeated. Such repeated measurements of the same source enable serendipitous variability searches, and indeed the current GALEX observations schedule is planned such to space out some of the deepest exposures, when possible, in order to maximize potential time-domain science, at no expense of additional exposure time-domain science. However, for the purpose of analyzing the content of the UV-source catalogs, we need to eliminate repeated measurements. Bianchi et al. (2010)'s catalogs of unique sources were constructed with the following criteria:

- They include only sources from observations when both detectors were on: occasionally one of the two detectors (most often FUV) was off, and including such data would bias statistical analyses of sources by FUV-NUV colors.

- They include sources within $0.5^{\circ}$ from the field center (a very conservative way to eliminate edge and rim artifacts, and to include good photometry). Such restriction eliminates also some true sources near the field's edge, however their astrometry and photometry would be less accurate (which may cause e.g. loss of sources in matching with other catalogs). Note that users interested in a specific small region of the sky may find it preferable to donwload the entire source list from a GALEX observation, and check manually for the quality of the sources near the edge (where elongated shapes of point sources lead to less accurate position and photometry).

- Duplicates were eliminated, considering a measurement 'duplicate' if closer than 2.5" to another source and from a different field/observation. In such cases, the measurement with the longest exposure time was retained, and in case of equal exposure, the one from the observation where the target was closer to the field center.

- The wider catalogs include all sources with photometric error in NUV merr NUV $\leq 0.5 \mathrm{mag}$, regardless of whether they are also detected in FUV.

With the above restrictions, out of $\sim 200$ million entries in the GALEX photometric database, the catalogs of 'unique' UV sources include 65.3M (AIS) and 12.6M (MIS) sources for GR5, with area coverages of 21435 (AIS) and 1579

\footnotetext{
${ }^{1}$ The catalogs are available from the author's web site at http://dolomiti.pha.jhu.edu/uvsky, and will soon be linked from the MAST database as value-added GALEX products, and as high-level science products.
} 
Fig. 1 Sky coverage of

GALEX fifth data release AIS

(blue) and MIS (green) surveys

in Galactic coordinates

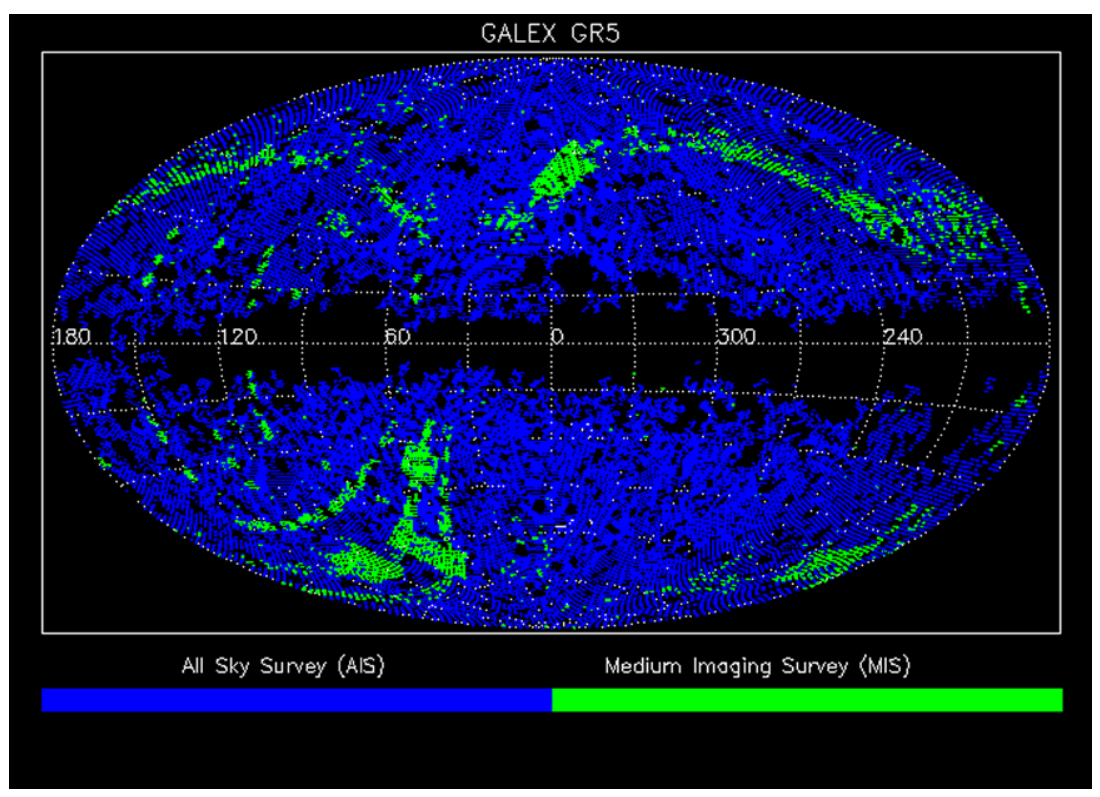

(MIS) square degrees (after excluding overlap, see Bianchi et al. 2010 for area calculation).

\subsection{Statistical properties}

In any analysis of astrophysical samples, it is important to understand completeness and selection biases. The typical exposure times of AIS and MIS are $\approx 100$ and 1500 s respectively (but see Fig. 2 of Bianchi et al. 2010 for actual exposure times distribution). AIS reaches typical $5 \sigma$ magnitudes of 19.9(FUV)/20.8(NUV) mag (AB magnitude system), and MIS of 22.6(FUV)/22.7(NUV) mag. Because UV wavelengths are very sensitive to extinction by interstellar dust, the actual depth for a given exposure time varies with location on the sky, the absorption being especially severe on the Milky Way (MW) disk (see Figs. 2 and 6 of Bianchi et al. 2010). Anyway, these general magnitude limits show that for equal exposure time, the FUV $5 \sigma$ limit is brighter than the NUV limit in the regime where the source counts are higher than the sky background (e.g. AIS), consistent with the fact that shorter wavelength photons are harder to detect. However, for an exposure of $\sim 1500 \mathrm{~s}$ the same magnitude limit is reached in both bands, because the sky background (about $10 \times$ higher countrate in NUV than in FUV, also owing to the zodiacal light contributing much of the NUV sky counts) becomes relevant relative to the source countrate. For longer integrations, the FUV magnitude limit may become slightly deeper than NUV.

This "cross-over" of FUV and NUV effective depth, at UV-magnitudes fainter than 23, due to relative sky $v s$ source counts, ${ }^{2}$ causes some interesting effects in sample selec-

${ }^{2}$ Using a typical countrate of $1000 \mathrm{cts} / \mathrm{s}$ for FUV, and $10000 \mathrm{cts} / \mathrm{s}$ for NUV (total counts, Morrissey et al. 2007), we estimate that the sky tions, which are further complicated by the different nature of UV sources in the sky predominating in different magnitude ranges. It is worth examining some of these trends which affect analyses based on source counts. The UVsource catalogs contain both stellar (MW) and extragalactic objects (galaxies and QSOs). In general, we may say both become much rarer at bluer FUV-NUV colors: hot stars are rarer than cooler, low-mass stars, and so on. This is reflected by the fact that, when we restrict the catalogs described in Sect. 2.1 to sources with photometric error $\leq 0.5 \mathrm{mag}$ in both FUV and NUV, we retain about 5.8M(AIS) and 3.0M(MIS) sources, i.e. a fraction of about $10-20 \%$ of the total NUVsources (Table 1).

It is interesting to further examine the variation of these average fractions (FUV detections over total NUV detections) with magnitude, and with Galactic latitude where the opposite effects (on source counts) of interstellar extinction and stellar density, both increasing towards the MW plane, combine. Figure 2 shows the fraction of sources detected in both FUV and NUV, over total NUV-detections, as a function of Galactic latitude. Although some latitudes have very little area coverage and poor statistics, there is a clear trend of the fraction to drop towards low latitudes. One may think at first that this reflects the interstellar extinction increasing at low latitudes, and affecting the FUV wavelengths more. This is mostly not the case; we recall that, although the selective-extinction $\mathrm{A}_{\lambda}$ does increase towards short wavelengths, the GALEX FUV-NUV color is almost reddening-free, for moderate extinction amounts and for "typical MW dust" $\left(\mathrm{R}_{V}=3.1\right)$, because the broad NUV

brightness over the approximate area of a point source is FUV $\sim 24.8$ 25.7 mag and NUV 23.6-24.5 mag. 
Table 1 UV source catalogs

\begin{tabular}{|c|c|c|c|c|c|c|}
\hline Latitude & $\operatorname{area}^{\mathrm{a}}$ & \# sources & \# sources & \# blue ${ }^{b}$ & \# blue ${ }^{b}$ & \# matches \\
\hline \multirow[t]{2}{*}{ [deg] } & [sq. deg.] & $\operatorname{err}_{\mathrm{NUV}}$ & errFUV,NUV & errFUV,NUV & errFUV,NUV & GSC-II \\
\hline & & $<0.5 \mathrm{mag}$ & $<0.5 \mathrm{mag}$ & $<0.5 \mathrm{mag}$ & $<0.3 \mathrm{mag}$ & $\left(\operatorname{rad}=3^{\prime \prime}\right)$ \\
\hline
\end{tabular}

\begin{tabular}{|c|c|c|c|c|c|c|c|}
\hline \multicolumn{8}{|l|}{ AIS } \\
\hline-90 & -80 & 249.3 & 826362 & 137185 & 26951 & 5422 & 440341 \\
\hline-80 & -70 & 714.0 & 2326390 & 378273 & 74772 & 14670 & 1241044 \\
\hline-70 & -60 & 1137.4 & 3602323 & 540957 & 107768 & 20979 & 1898093 \\
\hline-60 & -50 & 1490.2 & 4423999 & 569522 & 118193 & 24374 & 2378119 \\
\hline-50 & -40 & 1752.0 & 5170024 & 531483 & 118853 & 27564 & 2916337 \\
\hline-40 & -30 & 1910.2 & 5847401 & 494939 & 108340 & 28077 & 3541723 \\
\hline-30 & -20 & 1616.3 & 5290786 & 292725 & 69303 & 23488 & 3579275 \\
\hline-20 & -10 & 1015.9 & 3768865 & 145006 & 33457 & 15774 & 2847270 \\
\hline-10 & 0 & 211.3 & 550527 & 25800 & 3534 & 1845 & 341335 \\
\hline 0 & 10 & 349.5 & 1008773 & 51385 & 5728 & 2856 & 685642 \\
\hline 10 & 20 & 1487.0 & 4994835 & 231702 & 45038 & 18337 & 3583106 \\
\hline 20 & 30 & 2002.1 & 6152906 & 379885 & 83678 & 23873 & 4033350 \\
\hline 30 & 40 & 2115.2 & 5895410 & 437006 & 101768 & 22450 & 3514476 \\
\hline 40 & 50 & 1866.2 & 5456106 & 478963 & 112433 & 20434 & 3073487 \\
\hline 50 & 60 & 1480.0 & 4214819 & 446739 & 99119 & 17120 & 2317636 \\
\hline 60 & 70 & 1067.3 & 3036565 & 362872 & 77432 & 13284 & 1653003 \\
\hline 70 & 80 & 738.0 & 2064753 & 223993 & 49663 & 7470 & 1100107 \\
\hline 80 & 90 & 233.2 & 635448 & 80121 & 17250 & 2773 & 338143 \\
\hline-90 & 90 & 21434.8 & 65266292 & 5808556 & 1253280 & 290790 & 39482488 \\
\hline \multicolumn{8}{|l|}{ MIS } \\
\hline-90 & -80 & 29.4 & 244482 & 65865 & 8748 & 2162 & 91810 \\
\hline-80 & -70 & 59.3 & 506726 & 141739 & 17005 & 4041 & 181443 \\
\hline-70 & -60 & 257.4 & 2208613 & 623419 & 69126 & 17542 & 708703 \\
\hline-60 & -50 & 242.5 & 1877510 & 472030 & 62736 & 16935 & 677001 \\
\hline-50 & -40 & 150.5 & 1038628 & 226618 & 34806 & 9376 & 411794 \\
\hline-40 & -30 & 109.0 & 862096 & 162931 & 23612 & 6998 & 412264 \\
\hline-30 & -20 & 27.4 & 262708 & 38027 & 6433 & 2106 & 152042 \\
\hline-20 & -10 & 0.0 & 0 & 0 & 0 & 0 & 0 \\
\hline-10 & 0 & 0.0 & 0 & 0 & 0 & 0 & 0 \\
\hline 0 & 10 & 0.0 & 0 & 0 & 0 & 0 & 0 \\
\hline 10 & 20 & 5.8 & 43887 & 8626 & 997 & 304 & 24335 \\
\hline 20 & 30 & 89.1 & 630735 & 138846 & 17057 & 4454 & 302480 \\
\hline 30 & 40 & 210.3 & 1649769 & 356621 & 49273 & 13271 & 687277 \\
\hline 40 & 50 & 161.3 & 1348909 & 314522 & 42522 & 11320 & 545052 \\
\hline 50 & 60 & 175.7 & 1422683 & 341003 & 40449 & 10007 & 569293 \\
\hline 60 & 70 & 58.1 & 477213 & 123567 & 14439 & 3615 & 178931 \\
\hline 70 & 80 & 2.8 & 23953 & 6405 & 678 & 136 & 7754 \\
\hline 80 & 90 & 0.0 & 0 & 0 & 0 & 0 & 0 \\
\hline-90 & 90 & 1578.6 & 12597912 & 3020219 & 387881 & 102267 & \\
\hline
\end{tabular}

${ }^{\text {a}}$ For calculations of area coverage (taking into account overlap among fields) see Bianchi et al. (2010)

$\mathrm{b}_{\text {\# }}$ of GALEX sources with FUV-NUV $<-0.13$, with FUV and NUV photometric errors less than 0.5 mag (column 6 ) and 0.3 mag (column 7 ) 

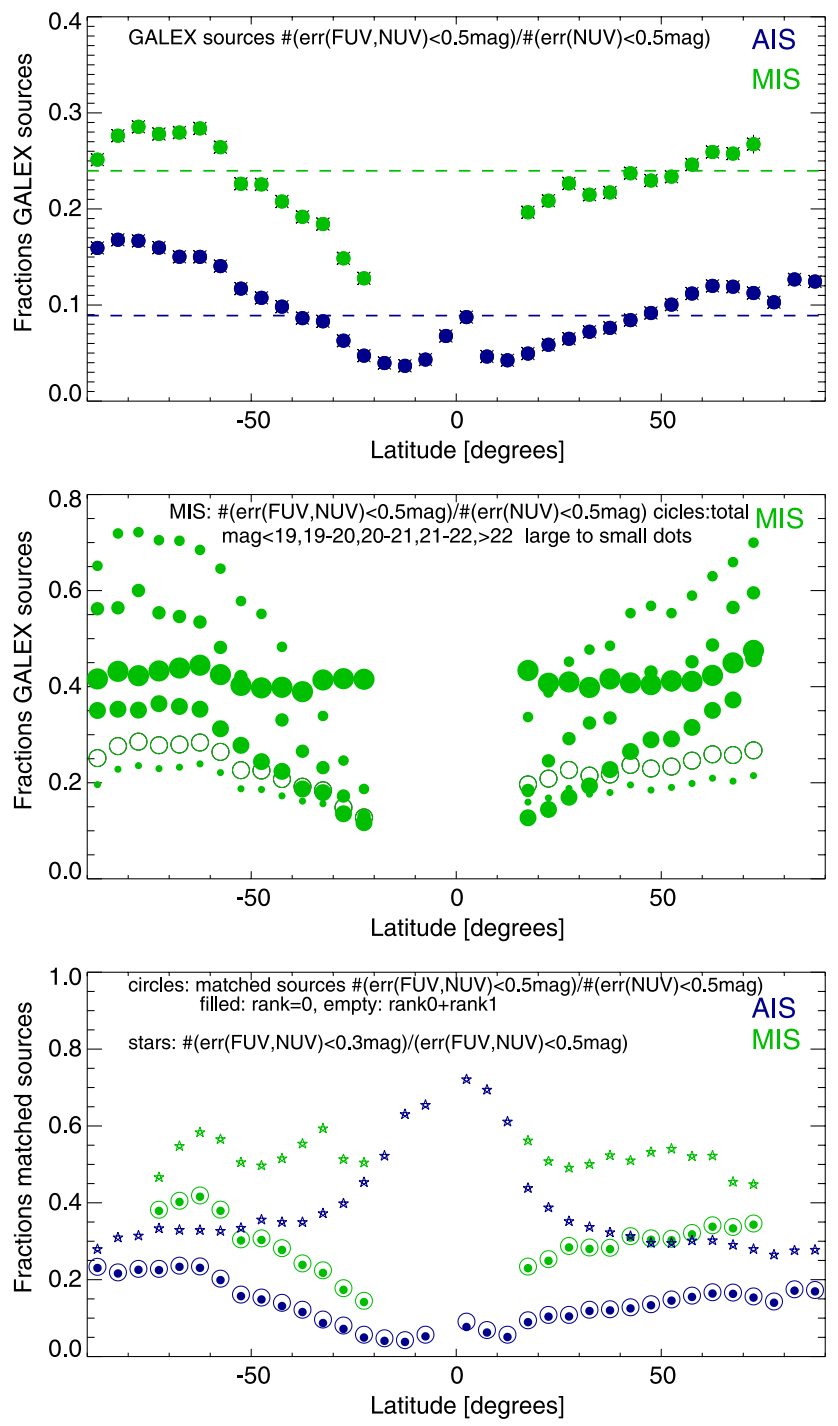

Fig. 2 Top: Number of GALEX sources (from the 'unique' GALEX source catalogs) with error cuts of $\leq 0.5 \mathrm{mag}$ in both FUV and NUV, over the number of sources with merr $_{\mathrm{NUV}} \leq 0.5 \mathrm{mag}$ (regardless of FUV detection), in bins of 5-degree Galactic latitude. Note a slight North-South asymmetry, but we recall that the sky coverage is not uniform with longitude (Fig. 1), and the MW population (and dust) have also a longitude dependence. Middle: The same for the MIS sample, divided in magnitude bins (and total, empty circles). Bottom: The same fraction for the matched GALEX-SDSS sources (dots), and the effect of progressive error cuts (from $\leq 0.5 \mathrm{mag}$ to $\leq 0.3 \mathrm{mag}$, in both bands, stars). MIS data are shown in green, AIS in blue

passband includes the strong $2175 \AA$ feature (see Bianchi 2011). On the other hand, the fraction is higher for MIS than it is for AIS, at all latitudes. This partly relates to the shallower depth of FUV at bright magnitudes (AIS) discussed earlier, and to the sky countrate affecting more the NUVband at faint magnitudes: both effects favour more relative FUV detections at faint magnitudes (for equal exposure time in FUV and NUV). The middle panel of Fig. 2 shows the same fractions, but separating the MIS sources in magnitude
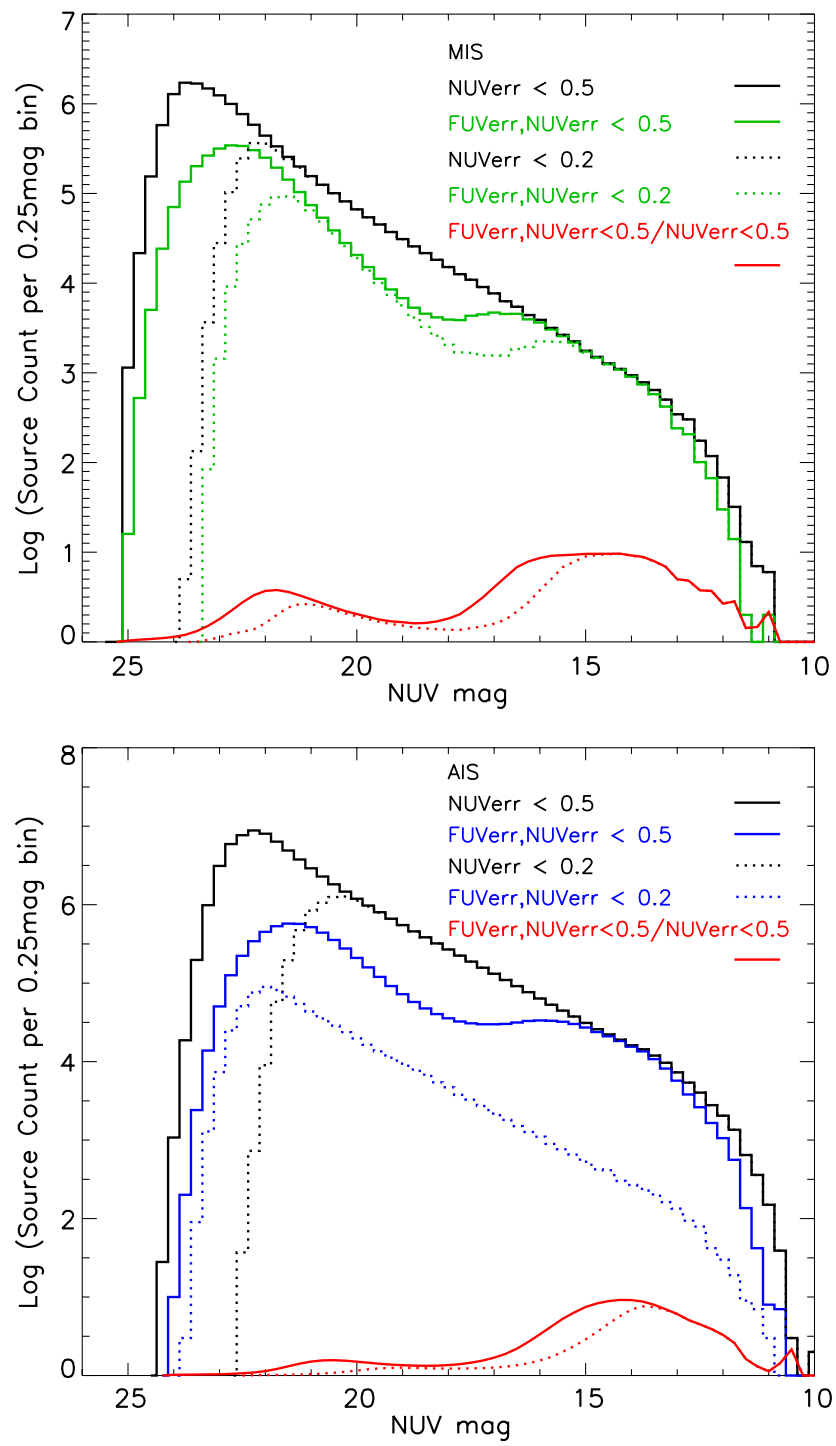

Fig. 3 NUV magnitude distribution of MIS sources: NUV-detections regardless of FUV detections (black), and sources with both FUV, NUV detections (green for MIS, blue for AIS). The red line is the ratio of the latter over the former. The dotted lines show the samples restricted by a 0.2 mag error cut

ranges. Sources brighter than $\sim 19$ th mag are not affected by latitude-dependent trends (but the MIS sky coverage does not extend to the MW plane, where the source counts steeply drop (see Fig. 2 of Bianchi et al. 2010), and this shows again the FUV-NUV color to be almost reddening-free. We recall that source counts increase towards fainter magnitudes, hence the MIS statistics is dominated by fainter sources. The faintest magnitude bin (the smallest dots) is the most numerous and drives the total statistics. The intermediate bins are harder to interpret, since the trends and absolute values of the fraction seem to reverse for sources fainter than 22nd mag. We think this is due to the previously mentioned sky-background countrate becoming significant, as well as (and perhaps more relevant) to different source classes being 

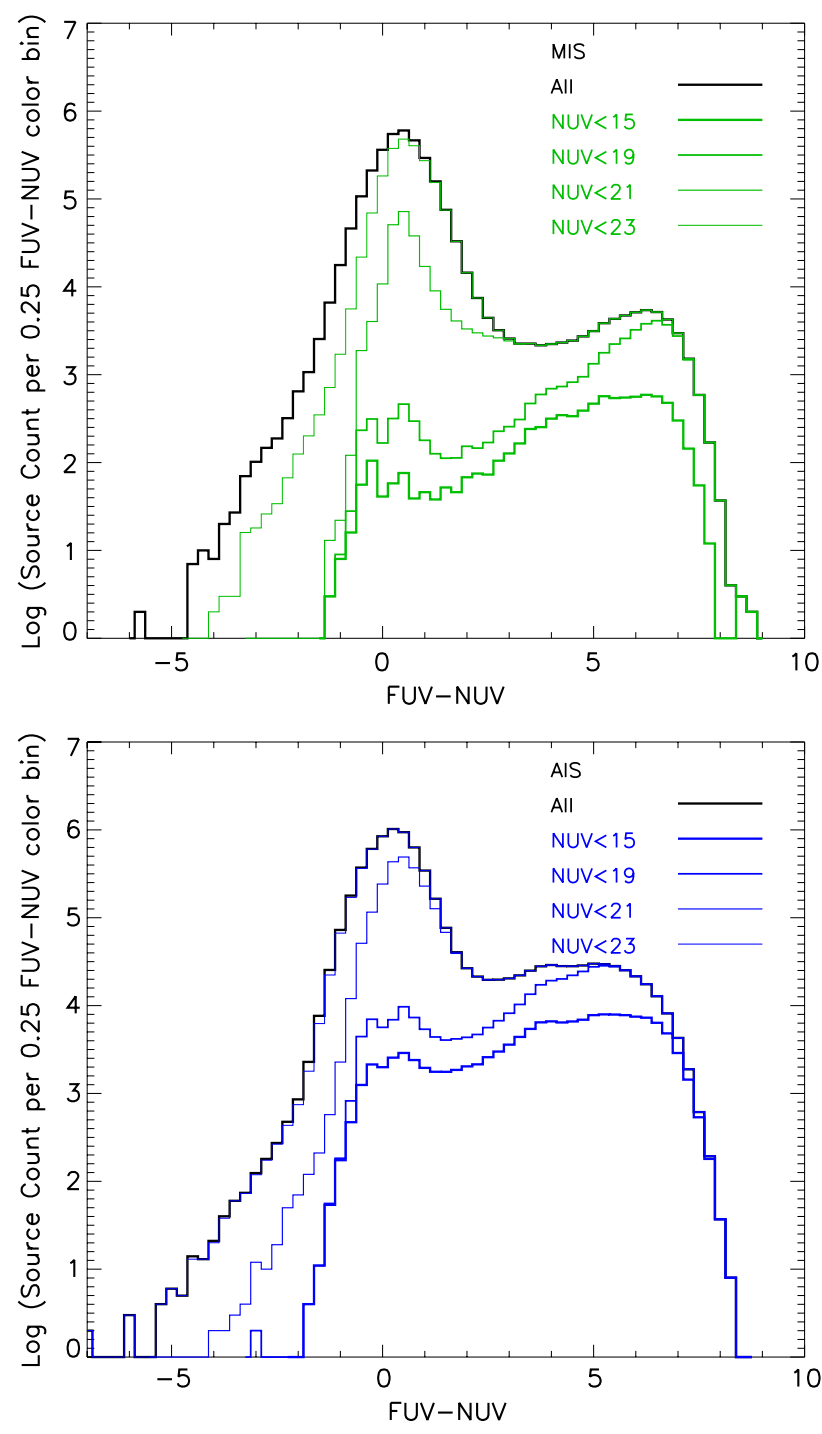

Fig. 4 FUV-NUV color distribution of MIS sources, with FUV, NUV error cuts of 0.5 mag and progressive magnitude cuts. The tail at unrealistic colors FUV-NUV $\ll-1$ consists of very faint sources with bad photometry, and perhaps some artifacts (which we expect to be irrelevant, due to the exclusion of the field's rim. Flags are included in the catalogs). Such tail is eliminated by error cuts, as expected

more or less numerous at various magnitudes, and having differing color distributions. For example, the FUV-NUV color of QSOs varies by as much as $0.9 \mathrm{mag}$ (up to $1.8 \mathrm{mag}$, depending on Ly $\alpha$ strength) between redshift 0 and 1 , due to Ly $\alpha$ going through the FUV passband between $z=0.1$ and 0.4 : Ly $\alpha$ is at the peak of FUV transmission for $z \sim 0.22$ and of NUV's transmission for $z \sim 0.8-1$ (see Bianchi et al. 2009a).

Figure 3 shows NUV-magnitude histograms of MIS and AIS sources, again comparing all NUV-detections with the number of sources significantly detected in both FUV and NUV (see Bianchi et al. 2010 for other histograms). It shows a marked magnitude dependence of the FUV-error cut ef-
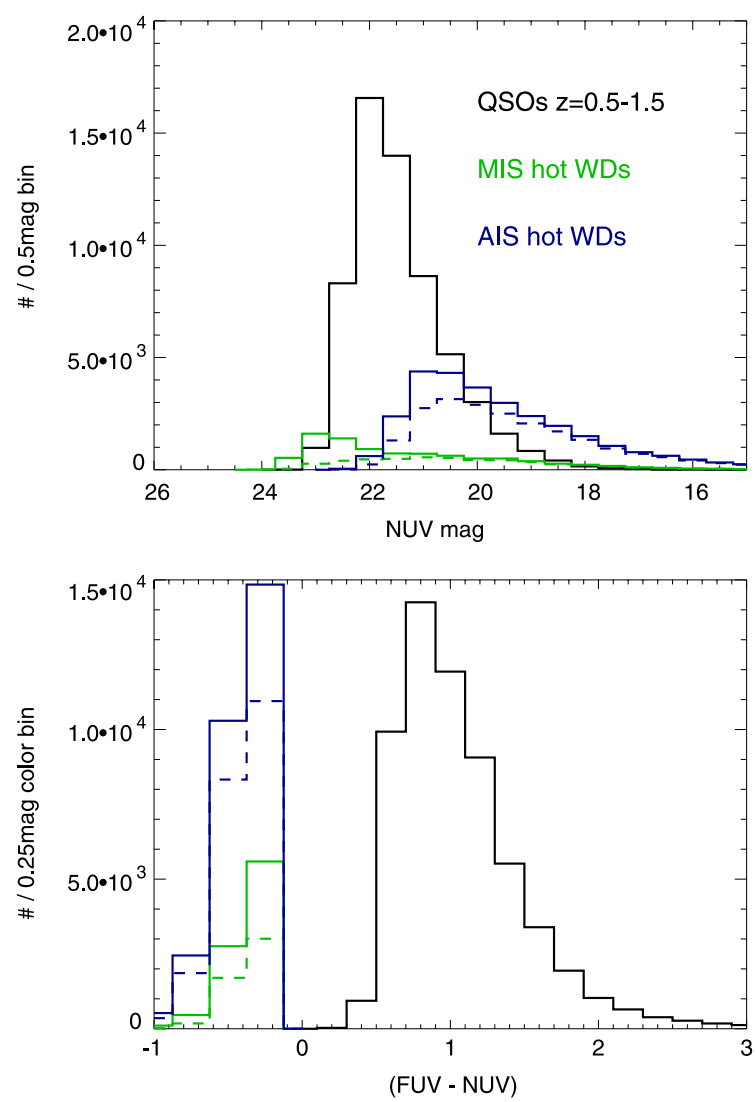

Fig. 5 Comparison of UV magnitude (top) and UV color distribution (bottom) of two very different specific samples of astrophysical objects: they have no overlap in color, owing to the intended sample selection, but the magnitude distributions overlap although with different relative shapes. Solid and dashed green/blue (MIS/AIS) lines are hot-star candidates from Bianchi et al. (2010), including and excluding binaries respectively (the 'binaries' sample has some contamination by extragalactic sources at faint magnitudes, the 'single' stellar sample has virtually no contamination). The QSO sample around redshift 1 is from Hutchings and Bianchi (2010)

fect, and confirms that the sample is dominated by different populations in different regimes, causing a double-peaked distribution when FUV error-cuts are imposed. This is illustrated also by Fig. 4, showing the FUV-NUV color distribution with progressive magnitude cuts (again it becomes double-peaked, the faint component being predominantly extragalactic). Finally, Fig. 5 shows two extremely different classes of astrophysical objects, hot WDs ( $\left.T_{\text {eff }}>18000 \mathrm{~K}\right)$, and QSOs with redshift range $0.5-1.5$, selected from these catalogs (and analyzed by Bianchi et al. 2010, and Hutchings and Bianchi 2010 respectively). These examples, and the ensemble of Figs. 2 to 5, explain why applying FUV and NUV error restrictions can modify (bias) extracted samples in different ways in differing magnitude regimes, which should be taken into account in flux-limited sample analyses. 


\section{Matched UV-optical source catalogs}

\subsection{Matched GALEX-SDSS catalogs}

In order to classify the UV sources by astrophysical classes, it is useful to match the UV catalogs with data at other wavelengths. Bianchi et al. (2010) matched their UV uniquesource catalogs analyzed in Sect. 2.1 with the SDSS (Sloan Digital Sky Survey) database, which provides five optical bands, $u g r i z$, with magnitude limits of $\approx 22.3,23.3$, 23.1, 22.3 and 20.8 ABmag respectively. Most UV sources have an SDSS counterpart, except for the bluest hottest WDs, where the MIS reaches deeper, and especially further out in the MW halo (see later). The bottom panel of Fig. 2 shows the statistical fractions of matched sources with both FUV and NUV detection over the total NUV-detected sources. These are very similar to the fractions in the original GALEX catalogs, since only the hottest sources (rare) are below SDSS detection.

Given the $\sim 3$ times higher spatial resolution of groundbased surveys, a GALEX source may have more than one optical counterpart within a given match radius: such cases are flagged in the catalog, because their GALEX magnitudes may be a composite of two nearby objects, hence their UV-optical colors misleading for physical classification. The matched catalogs were constructed with a match radius of $3^{\prime \prime}$, which was found a good compromise to minimize spurious additional matches, without unnecessarily losing true matches. Bianchi et al. (2010) estimated an incidence of spurious matches (positional coincidence) of the order of few percent, also latitude-dependent, as can be expected.

\subsection{Matched GALEX-GSC-II Catalog}

Although the SDSS has depth almost comparable to MIS, and sufficient to match all AIS sources, its restricted area coverage limits the possibility of analyzing the MW structure of UV-optical sources. Given the much larger sky coverage of GALEX AIS than SDSS, we also matched the shallower, wide-area AIS catalog with GSC-II (Lasker et al. 2008), in order to explore a longitude dependence of the local stellar populations (MW thin and thick disk). GSC2 provides up to three optical magnitudes, $B_{J}, R_{F}$, and $I_{N}$. The $B_{J} \sim 22$ mag "limit" for GSC-II quoted by Lasker et al. (2008) probably refers to the faintest end of the magnitudes range. From their Fig. 3 we infer a calibrated magnitude limit of $B_{J} \sim 20 \mathrm{mag}$ at a $80-90 \%$ completeness, which drops to less than $50 \%$ at $B_{J} \sim 21 \mathrm{mag}$. The limit is brighter (or, the completeness lower) for the other bands, and the catalog includes only objects detected and measured at least on one IIIaF (R) or IIIaJ (B) or IV-N (I) plate. Therefore, we expect to have matches fairly complete down to $B \sim 20$ or so, but it is not possible to assess a consistent limit of GSC-II

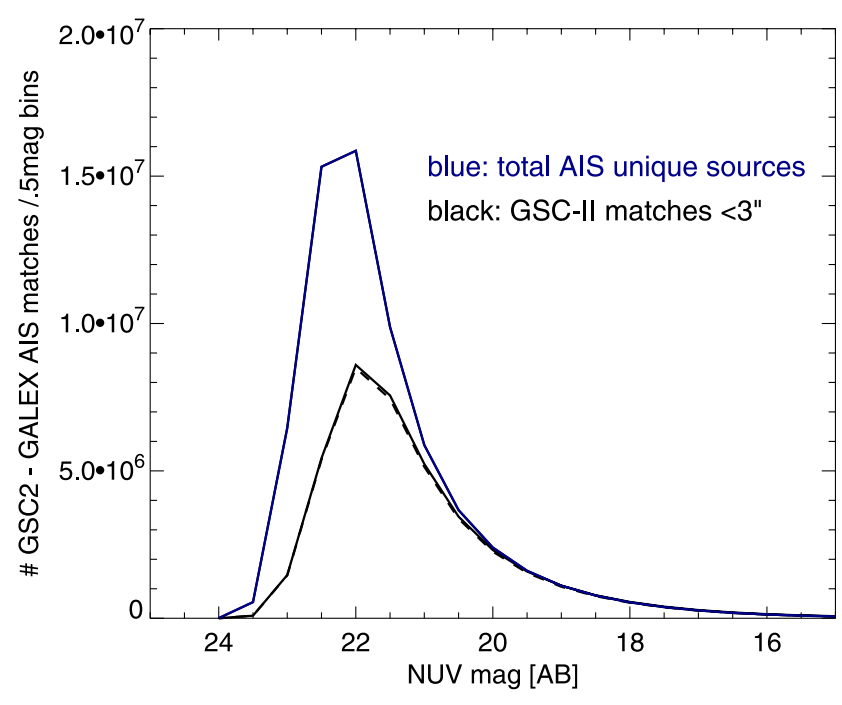

Fig. 6 NUV magnitude distribution of AIS sources matched to GSC-II (black solid line: all matches, black dashed-line: matches with only one optical counterpart). The blue line is the total AIS unique-source catalog

across the sky. We find a match in the GSC-II for about $60 \%$ of our AIS unique-UV sources (match radius $=3^{\prime \prime}$ ), with a slight variation among high latitudes $(\sim 55 \%$ and $54 \%$ for $90-45^{\circ}$ North and South) and low latitudes ( $\sim 64 \%$ and $65 \%$ for $0-45^{\circ}$ North and South; for comparison, with a match radius of $5^{\prime \prime}$ these figures become $\sim 73 \%$ and $74 \%$, but the increase are mostly multiple and spurious matches, hence the purity decreases).

Figure 6 shows that indeed GSC-II incompleteness sets in at NUV $\sim 20$ in the matched catalog. One must recall that a given limit in one photometric band affects differently sources of different colors. For example, the limit in the optical catalogs causes the loss of the hottest sources first, in a given UV-magnitude bin.

\subsection{Matches with other catalogs}

We are in the process of matching Bianchi et al. (2010) UV source catalogs with other databases, such as 2MASS, USNO-B, and deeper optical catalogs that will become available. These catalogs will be added to the website as soon as they are validated and characterized.

\section{Selection of hot MW stars and analysis with Milky Way models}

\subsection{Hot stars sample}

One of the unique advantages of a FUV-NUV color is the selection of hot stellar sources (see Bianchi 2007). The GALEX-SDSS matched catalogs contain 28 319(AIS) and 
9028 (MIS) matched sources with FUV-NUV $<-0.13$ (including only sources with merr $_{\mathrm{FUV}}$, merr $\mathrm{NUV}<0.3 \mathrm{mag}$, and excluding sources with multiple optical matches); this color cut corresponds to stellar $T_{\text {eff }} \gtrsim 18000 \mathrm{~K}$, the exact value varying with gravity (see Fig. 7 of Bianchi et al. 2010). An additional color cut of NUV-r $>0.1$ isolates binaries with a hot star, and some intruding QSOs (more numerous at faint magnitudes), see Fig. 5 of Bianchi et al. (2010) and Figs. 3-5 of Bianchi (2009).

The hot-star sample includes mostly evolved stellar objects: post-AGB stars crossing the HR diagram towards higher $T_{\text {eff' }}$ ' at constant luminosity (becoming planetary nebulae nuclei), elusive because they evolve very fast, on time-scales of the order of $10^{3}-10^{5}$ years, and hot white dwarfs which are cooling and fading in luminosity. These evolve on longer timescales, but at significantly fainter luminosities. Both are elusive at all wavelengths except the UV.

\subsection{Analysis. The initial-final mass relation}

Bianchi et al. (2010) analyzed the distribution of hot star density as a function of MW latitude, computing MW models with the TRILEGAL stellar population synthesis code (Girardi et al. 2005). The geometry of the MW components in TRILEGAL was calibrated from star counts in a local sample from the Hipparcos catalog, plus the shallow all-sky 2MASS, and a few deep surveys. Reddening is taken into account by distributing along each line of sight the total $\mathrm{E}$ (BV) estimated from the Schlegel et al. (1998) maps, assuming an exponential dust layer with scale height $h_{z}^{\text {dust }}=110 \mathrm{pc}$.

Figure 7 shows model predictions for thin disk, thick disk, and halo stellar components, as well as the total predicted counts, for a sample latitude range. Several ingredients in the model calculations, such as the WD birthrate, the assumed MW extinction model, and the geometry affect model predictions. A most critical assumption for modeling hot-WD counts is the Initial-Final Mass Relation (IFMR), to which our data are particularly sensitive. It was therefore explored first. An IFMR biased towards lower final masses, such as that of Weidemann (2000), produces a better match of the model calculations with the observed stellar counts than e.g. the Marigo and Girardi (2007) IFMR, which was calibrated from AGB stars analysis, but largely overpredicts faint hot-WD star counts (Fig. 7). This trend is seen at all Galactic latitudes (Bianchi et al. 2010): model predictions with an IFMR favouring low final masses match well the observed stellar counts at bright magnitudes, and intermediate latitudes, but are somewhat discrepant at fainter magnitudes, where the samples are however affected by the biases discussed in Sect. 2.2. Constraining the IFMR for intermediatemass stars is relevant for understanding the yield of chemical elements (see Bianchi et al. 2010 for a discussion).
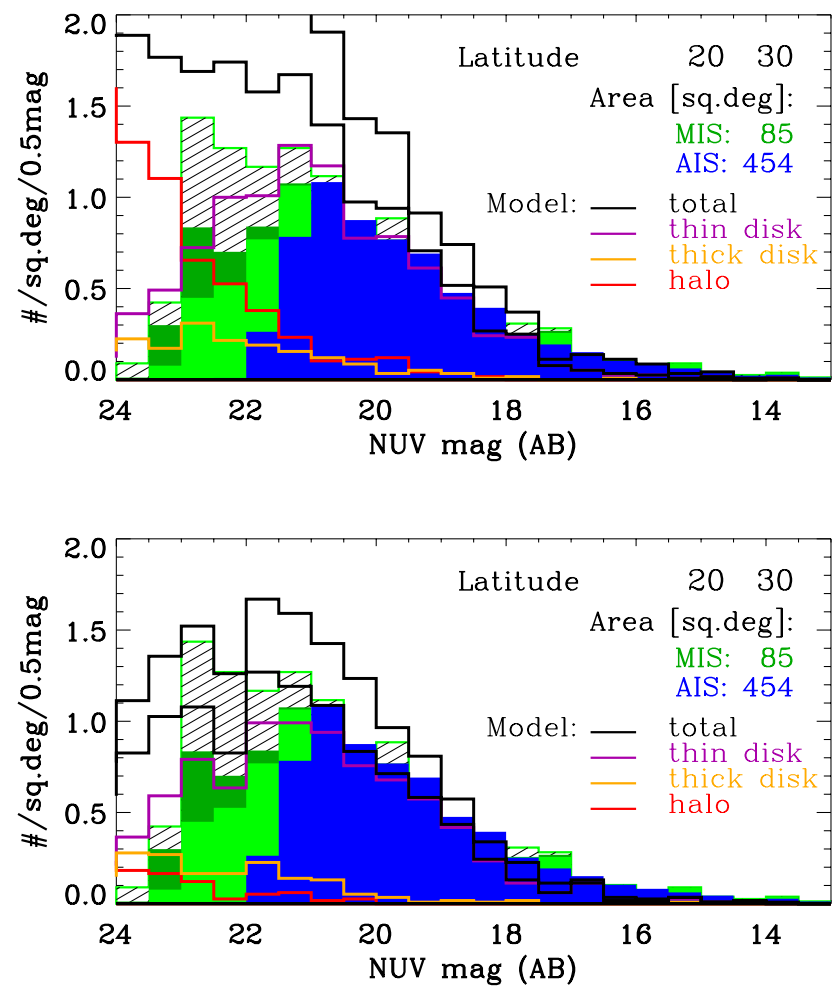

Fig. 7 Density of hot-WDs (FUV-NUV $<-0.13$ ) at latitudes $b=20-30^{\circ} \mathrm{N}$ : blue from AIS, green from MIS. The light-green histograms are "single" hot-WDs from MIS GALEX-SDSS matches, the dark-green are GALEX hot MIS sources within the SDSS footprint but with no SDSS match. Dashed-green histograms are matched GALEX-SDSS hot-WDs including single and binaries (the latter sample being contaminated by extragalactic objects). Model predictions (lines, for halo, thin and thick disk components, and total) are computed assuming the Marigo and Girardi (2007) IFMR (top) and Weidemann (2000) IFMR (bottom). Thick disk and halo counts become significant at magnitudes fainter than $\sim 19$, and $\sim 20$, for these two IFMR respectively. The higher of the two black lines includes counts for both single and binary stars, the lower black line only single stars (corresponding to the filled histograms of the data counts)

The brightest (and complete) sample mostly contains hot WDs in the thin disk at distances up to $\sim 1 \mathrm{kpc}$. The faintest part of the sample may contain a significant fraction of stars more distant than $2 \mathrm{kpc}$, including hot WDs in the thick disk and halo: these components become significant at UV magnitudes $\lesssim 21$ (Fig. 7) and require UV surveys at the depth of MIS or fainter, and optical surveys deeper than SDSS.

Acknowledgements GALEX (Galaxy Evolution Explorer) is a NASA Small Explorer, launched in April 2003. We gratefully acknowledge NASA's support for construction, operation, and science analysis of the GALEX mission, developed in cooperation with the Centre National d'Etudes Spatiales of France and the Korean Ministry of Science and Technology. Data presented in this paper were obtained from the Multimission Archive at the Space Telescope Science Institute (MAST). STScI is operated by the Association of Universities for Research in Astronomy, Inc., under NASA contract NAS526555. L.B., J.H. \& B.E. acknowledge partial support from NASA grant NNX07AJ47G, A.Z. from CNPq-MCT/Brazil, L.G. from ASI- 
INAF I/016/07/0. We are very grateful to Patrick Morrissey and Karl Forster for useful discussions.

Open Access This article is distributed under the terms of the Creative Commons Attribution Noncommercial License which permits any noncommercial use, distribution, and reproduction in any medium, provided the original author(s) and source are credited.

\section{References}

Bianchi, L., et al.: Mon. Not. R. Astron. Soc. (2010), doi:10.1111/ j.1365-2966.2010.17890.x

Bianchi, L.: Astrophys. Space Sci. 320, 11 (2009)

Bianchi, L., et al.: Astron. J. 137, 3761 (2009a)

Bianchi, L., Efremova, B., Herald, J., et al.: In: Van Steenberg et al. (eds.) Future Directions in Ultraviolet Spectroscopy. AIPC, vol. 1135 , p. $326(2009 b)$
Bianchi, L.: In: Gomez de Castro, A., Barstow, M. (eds.) UV Astronomy: Stars from Birth to Death, p. 65 (2007). ISBN 978-84-7491852-6

Bianchi, L., et al.: Astrophys. J. Suppl. Ser. 173, 659 (2007)

Bianchi, L., et al.: Astrophys. J. 619, L27 (2005)

Bianchi, L.: (2011), this book

Conti, A., Bianchi, L., Shiao, B., et al.: (2011), this book

Girardi, L., et al.: Astron. Astrophys. 436, 895 (2005)

Hutchings, J., Bianchi, L.: Astron. J. 140, 1987 (2010)

Lasker, B., et al.: Astron. J. 136, 735 (2008)

Marigo, P., Girardi, L.: Astron. Astrophys. 469, 239 (2007)

Martin, D.C., et al.: Astrophys. J. Lett. 619, L1 (2005)

McCandliss, S.R., et al.: Proc. SPIE 4, 5488-6117 (2005)

Morrissey, et al.: Astrophys. J. Suppl. Ser. 173, 682 (2007)

Schlegel, D.J., et al.: Astrophys. J. 500, 525 (1998)

Shustov, B., Sachkov, M., Gomez de Castro, A.I., et al.: Astrophys. Space Sci. 320, 187 (2009)

Weidemann, V.: Astron. Astrophys. 363, 647 (2000) 